\title{
The role of surface glycoconjugates in Leishmania midgut attachment examined by competitive binding assays and experimental development in sand flies
}

\author{
LUCIE JECNA ${ }^{1}$, ANNA DOSTALOVA ${ }^{1}$, RAY WILSON ${ }^{2}$, VERONIKA SEBLOVA ${ }^{1}$, \\ KWANG-POO CHANG ${ }^{3}$, PAUL A. BATES ${ }^{4}$ and PETR VOLF ${ }^{1}$ P \\ ${ }^{1}$ Department of Parasitology, Faculty of Science, Charles University, Vinicna 7, 12844 Prague 2, Czech Republic \\ ${ }^{2}$ Centre for Immunity, Infection and Evolution, Institute for Immunology and Infection Research, School of Biological \\ Sciences, University of Edinburgh, Edinburgh, UK \\ ${ }^{3}$ Department of Microbiology/Immunology, Chicago Medical School/Rosalind Franklin University, North Chicago, Illinois \\ 60064, USA \\ ${ }^{4}$ Division of Biomedical and Life Sciences, School of Health and Medicine, Lancaster University, Lancaster LA1 4YQ, UK
}

(Received 15 November 2012; revised 5 February and 5 March 2013; accepted 6 March 2013)

SUMMARY

Binding of promastigotes to the sand fly midgut epithelium is regarded as an essential part of the Leishmania life cycle in the vector. Among Leishmania surface molecules putatively involved in attachment to the sand fly midgut, two GPI-anchored molecules are the most prominent: lipophosphoglycan (LPG) and promastigote surface protease gp63. In this work, we examined midgut attachment of Leishmania lines mutated in GPI-anchored molecules and compared results from 2 different techniques: in vivo development in sand flies and in vitro competitive binding assays using fluorescently labelled parasites. In combination with previous studies, our data provide additional support for (1) an LPG-independent parasitebinding mechanism of Leishmania major within the midgut of the permissive vector Phlebotomus perniciosus, and provide strong support for (2) the crucial role of L. major LPG in specific vector Phlebotomus papatasi, and (3) a role for Leishmania amazonensis gp63 in Lutzomyia longipalpis midgut binding. Moreover, our results suggest a critical role for GPI-anchored proteins and gp63 in Leishmania mexicana attachment to L. longipalpis midguts, as the wild type (WT) line accounted for over $99 \%$ of bound parasites.

Key words: phlebotomine sand flies, Leishmania, lipophosphoglycan, zinc protease gp63.

\section{INTRODUCTION}

Leishmaniasis is a vector-borne disease transmitted by phlebotomine sand flies. Development of Leishmania in female sand flies starts upon the release of amastigotes from infected macrophages ingested by the fly during the blood meal, which then transform into motile procyclic promastigotes with short flagella (reviewed by Bates and Rogers, 2004; Kamhawi, 2006). These forms grow within the blood meal surrounded by a semi-permeable peritrophic matrix. After the peritrophic matrix begins to become degraded (Sadlova and Volf, 2009) procyclic promastigotes transform into highly motile elongated nectomonad forms, whose function is to escape from the endoperitrophic space and anchor themselves to the midgut epithelium. Such binding of promastigotes to the midgut epithelium is regarded as an essential part of the life cycle in the sand fly vector, enabling the parasites to persist beyond the initial blood-meal phase. Laboratory studies examining the

\footnotetext{
* Corresponding author. Department of Parasitology, Charles University, Vinicna 7, 12844 Prague 2, Czech Republic. E-mail: volf@cesnet.cz
}

development of different Leishmania in a range of sand fly species have revealed that so-called specific (restricted or non-permissive) vectors, for example, Phlebotomus papatasi and Phlebotomus sergenti, support the growth of only the parasites they normally transmit in nature, while other sand fly species, for example, Lutzomyia longipalpis, support the growth of various Leishmania spp. and are called permissive vectors. These two groups of sand flies differ in their mechanisms of parasite attachment to the midgut epithelium (Volf and Myskova, 2007).

Among Leishmania surface molecules, putatively involved in attachment to sand fly midgut, two GPIanchored molecules are prominent: promastigote surface protease gp63 and lipophosphoglycan (LPG). Gp63 (also known as leishmanolysin) is a zinc proteinase of molecular mass $58-65 \mathrm{kDa}$ with a wide substrate specificity and $\mathrm{pH}$ optimum (Yao et al. 2003). LPG is a major surface glycoconjugate which has been implicated in a wide range of functions both in the mammalian host and vector, including adhesion to sand fly midgut epithelium lectins (Kamhawi et al. 2004). Species-specific differences in the structure of LPG are thought to be at least partially responsible for the variation 
in sand fly vectorial competence for different Leishmania species (Sacks and Kamhawi, 2001).

Two major techniques for studying Leishmania attachment in the sand fly midgut have been used to date: in vivo development in sand flies and in vitro binding assays. Recently we developed an improved in vitro method, the competitive binding assay, where 2 promastigote populations are labelled with different fluorescent dyes and compete for binding to dissected sand fly midguts (Wilson et al. 2010). The advantage of this assay is a direct microscopic examination of a mixture of 2 differentially stained parasite populations bound to individual sand fly midguts dissected from flies at an appropriate time post-blood feeding. Assays are performed in Grace's Insect Medium in which guts do not change their structural integrity and remain amenable to manipulation for several hours. Parasites are counted from one microscope field of view per gut instead of homogenizing the whole gut and counting the released parasites on a haemocytometer, which, due to differences in sizes of the guts and quality of dissections, introduces a significant source of variation. In this study, we have used parasites mutated in LPG and gp63 and examined their role in parasite attachment to midguts of various sand fly species using both competitive in vitro binding assays and in vivo development in the sand fly midgut.

\section{MATERIALS AND METHODS}

\section{Parasites and sand flies}

The following parasite isolates were used in this study: Leishmania major LV39 clone 5 (MRHO/SU/ 1959/Neal P) wild type (WT) and an isogenic LPGdeficient mutant $\left(\operatorname{lpg} 1^{-/-}\right)$, which specifically lacks LPG through deletion of the LPG1 galactofuranosyltransferase gene required for synthesis of the LPG core (Späth et al. 2000); 2 similar lines of Leishmania infantum chagasi (MHOM/BR/76/M4192): WT and lpg $1^{-/-}$, generated for the purposes of this study (see below); 2 lines of Leishmania amazonensis LV78 (MPRO/BR/72/M1845): transfectants P6.5 (control) and P6 5/1.9R down-regulated for expression of gp63 (Chen et al. 2000); and 3 lines of Leishmania mexicana (MNYC/BZ/62/M379): WT, LPG (Ilg et al. 2001) and $g p^{-/-}$lacking expression of all cell surface GPI-anchored proteins (Hilley et al. 2000). Leishmania infantum $\operatorname{lpg} 1^{-1-}$ was generated essentially as described in Späth et al. (2000) by sequential removal of both alleles and replacement with blasticidin and hygromycin resistance genes (Brooks et al. 2000).

All parasites were maintained as promastigote forms at $26^{\circ} \mathrm{C}$ on Medium 199 supplemented with $20 \%$ fetal calf serum (Gibco) and gentamicin $\left(25 \mu \mathrm{g} \mathrm{mL} \mathrm{m}^{-1}\right)$. For the mutated lines, selection antibiotics were added to the culture medium as follows: hygromycin $\left(15 \mu \mathrm{g} \mathrm{mL} \mathrm{m}^{-1}\right)$ and puromycin $\left(11 \mu \mathrm{g} \mathrm{mL} \mathrm{m}^{-1}\right)$ for the L. major $\operatorname{lpg} 1^{-/-}$mutant; blasticidin $\left(50 \mu \mathrm{g} \mathrm{mL} \mathrm{mL}^{-1}\right)$ and hygromycin $\left(50 \mu \mathrm{g} \mathrm{mL}^{-1}\right)$ for the $L$. infantum chagasi lpg $1^{-/-}$

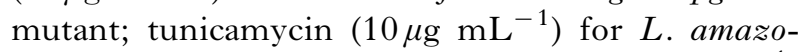
nensis transfectants; and nourseothricin $\left(25 \mu \mathrm{g} \mathrm{mL}^{-1}\right)$ and puromycin $\left(10 \mu \mathrm{g} \mathrm{mL} \mathrm{m}^{-1}\right)$ for the L. mexicana gpi $8^{-/-}$mutant.

Laboratory colonies of 4 sand fly species were used: Phlebotomus papatasi (origin from Turkey), $P$. perniciosus (Spain), P. sergenti (Israel) and Lutzomyia longipalpis (Jacobina, Brazil). Sand flies were maintained at $26^{\circ} \mathrm{C}$ on $50 \%$ sucrose under conditions described previously (Volf and Volfova, 2011).

\section{Leishmania development in sand flies}

Sand fly females were fed through a chick-skin membrane on heat-inactivated rabbit blood containing $10^{6}$ promastigotes per $\mathrm{mL}$ of blood. Prior to sand fly experimental feeding parasites were washed by centrifugation and re-suspended in saline solution. Engorged sand flies were separated and maintained at $26^{\circ} \mathrm{C}$ and dissected 2, 4 and 8 days after the bloodmeal. Parasite loads were graded as described previously by Cihakova and Volf (1997) as light (<100 parasites per gut), moderate (100-1000 parasites per gut) and heavy (>1000 parasites per gut). Data from 2 experiments were pooled and evaluated statistically by means of $\chi^{2}$ test using the S-PLUS 2000 program.

\section{Competitive in vitro binding assay}

Lutzomyia longipalpis, P. perniciosus, P. papatasi and $P$. sergenti midguts were dissected in Grace's Insect Medium (GIM, Sigma) 4-6 days after bloodfeeding. By this time all species had defecated blood meal remnants. Midguts were carefully opened longitudinally using a fine glass needle and the assays performed as described previously (Wilson et al. 2010). Briefly, 4-day-old cultures of promastigotes parasites, in which the nectomonads and leptomonads were the predominant forms, were labelled for $1 \mathrm{~h}$ at $26^{\circ} \mathrm{C}$ with $0 \cdot 04 \%(\mathrm{v} / \mathrm{v})$ Syto 21 Green or $0 \cdot 2 \%$ Syto40 Blue fluorescent dyes (Invitrogen), washed 3 times in M199 medium (Sigma) and twice in GIM, then re-suspended in GIM at a cell density of $10^{8}$ cells $\mathrm{mL}^{-1}$. Each midgut was placed in the well of a microscope chamber slide and incubated at RT for $45 \mathrm{~min}$ in a humid chamber with a $10 \mu \mathrm{L}$ mixture of green- and blue-fluorescent parasites. The guts were then individually washed 5 times by placing them in successive drops of GIM and placed in $2 \mu \mathrm{L}$ of cooled CyGel (Biostatus Limited) on a microscope slide, carefully flattened and mounted in Prolong Gold anti-fade reagent (Invitrogen). Bound parasites 
Table 1. Competitive binding assays of various Leishmania species and mutants defective in expression of surface molecules to the midguts from permissive and specific sand fly species

(The numbers are mean \pm standard deviation (S.D.) bound/midgut field of view from all examined midguts for a given vector species, where $n$ is the number of midguts analysed and the figure in parentheses is the total number of parasites counted. The result from each set of comparisons is displayed as a\% of the mutant parasites in a $1: 1$ mixture of mutant and wild type (WT). Equal binding of the mutant with WT will produce a $\%$ value close to $50 \%$; values above this would indicate preferential binding of the mutant, values below indicate preferential binding of the WT.)

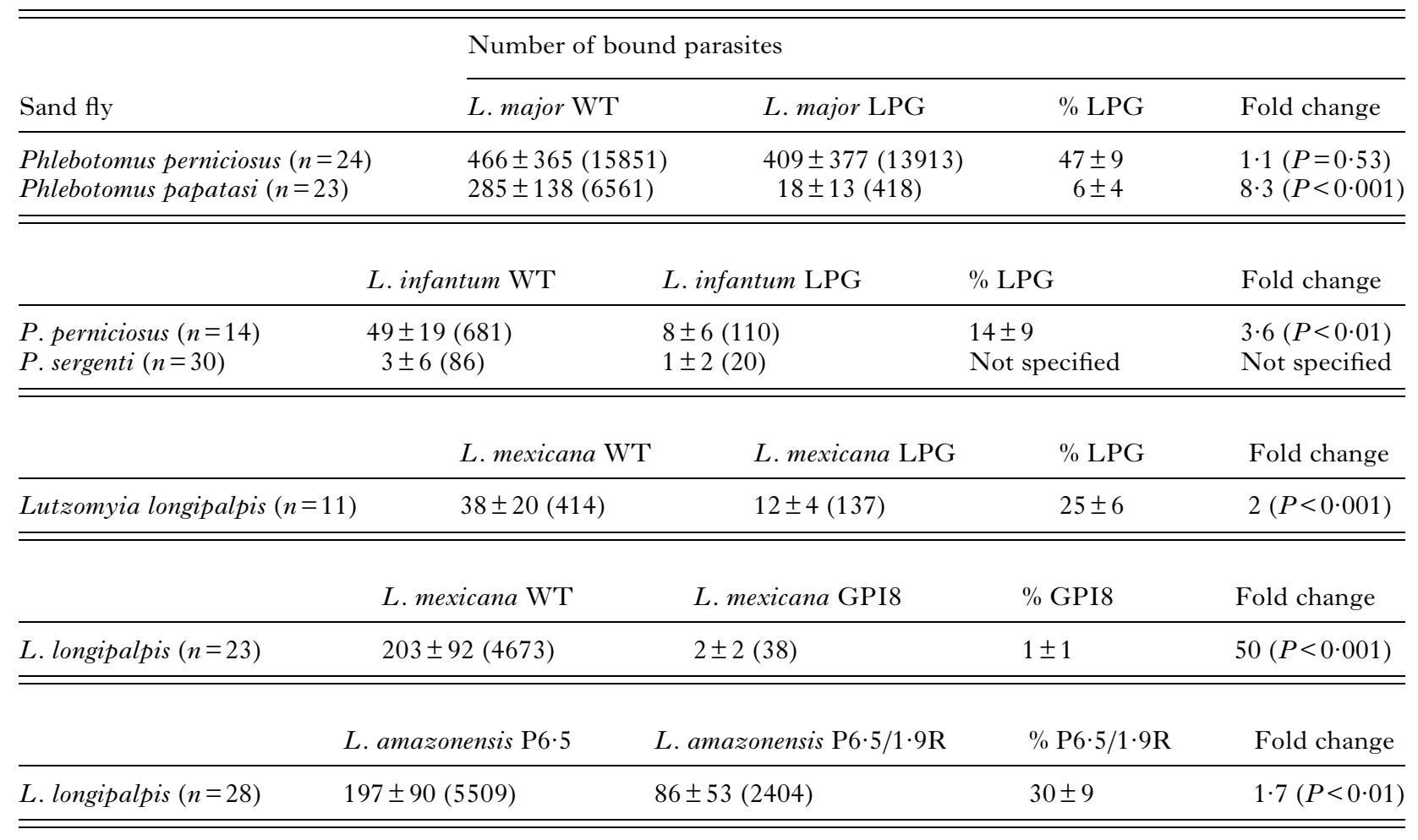

of both populations were visualized using a fluorescence microscope at $400 \times$ magnification with a CFP/YFP-A filter set (Olympus). The numbers of bound parasites from each population were counted in 1 field of view per midgut. Each experiment was repeated with the colour labelling of the parasites switched and each pair of comparisons was performed at least twice. The difference between bound parasites of 2 lines was evaluated statistically by means of the ANOVA test.

\section{RESULTS}

Binding of L. major LPG mutant to P. perniciosus and P. papatasi midguts

The role of LPG in the binding of $L$. major to a permissive vector was studied by incubation of the L. major LPG-mutant and WT control with midguts of $P$. perniciosus; a similar comparison was made using the natural and specific vector of $L$. major, P. papatasi (Table 1). As expected, the LPG-mutant was not able to bind to midguts of $P$. papatasi and was strongly out-competed by WT parasites, the mutant accounted for only $6 \%$ of the total bound parasites in competition with the WT $(P<0.001)$. On the other hand, using midguts of the permissive vector
$P$. perniciosus the LPG-mutant bound equally well as L. major WT (47\% of bound parasites were LPGnegative, showing no significant difference from that of the WT, $P=0 \cdot 53$ ).

Binding of L. infantum LPG mutant parasites and their development in $\mathrm{P}$. perniciosus and $\mathrm{P}$. sergenti

The existence of a non-LPG-mediated attachment mechanism that can support the development of L. major in a number of (unnatural) permissive vectors does not directly prove that such a mechanism is also used by parasites that are normally transmitted by such sand flies. To test this hypothesis directly, L. infantum LPG-mutants were generated and their abilities to develop in $P$. perniciosus and bind to midguts of this sand fly were investigated. Interestingly, the $L$. infantum LPG-mutant developed relatively poorly in $P$. perniciosus (Fig. 1). On days 4 and 8 post blood meal, LPG-mutants were present only in $50-60 \%$ of females and less than $15 \%$ of them had high parasite density in the midgut and colonized stomodeal valve. The differences in the positivity rate (\% of Leishmania-positive females) and parasite loads of W'T and LPG-negative parasites were statistically highly significant $(P<0 \cdot 01)$. Competitive binding assays also revealed that $L$. infantum LPG-negative 


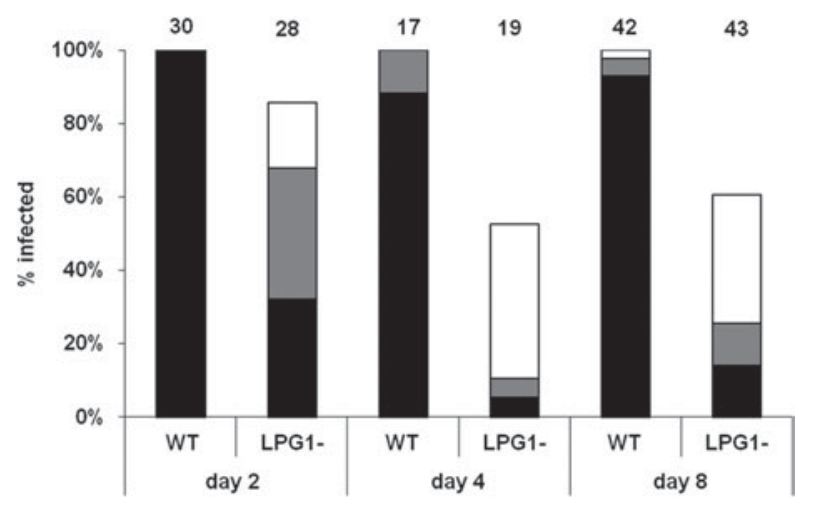

Fig. 1. Development of Leishmania infantum LPGmutant in Phlebotomus perniciosus. P. perniciosus females were fed with $L$. infantum wild type (WT) or mutants lacking LPG $\left(\operatorname{lpg} 1^{-/-}\right)$. Day 2 and Day 4 dissections were before defecation, Day 8 dissections were after defecation. Parasite loads were classified into three categories: heavy (more than 1000 parasites per gut) - black bars; moderate (100-1000 parasites per gut) - grey bars; light (1-100) white bars. Numbers above the bars indicate the number of dissected females.

parasites competed poorly against the WT control for binding to $P$. perniciosus midguts, representing only $14 \%$ of the total population $(P<0 \cdot 01$; Table 1$)$.

To explore the properties of the L. infantum LPG-mutant further, its ability to develop in and bind to midguts of the specific vector P. sergent $i$ was investigated. On day 2 post blood feeding, both mutant and WT lines, initially produced moderate or heavy parasite loads in the abdominal midgut. However, after defecation, on days 5 and 8 , no parasites were found in any dissected flies. Consistent with this finding, neither $L$. infantum WT nor LPG-negative parasites were found to bind to $P$. sergenti midguts in significant numbers (Table 1 ).

\section{Binding of L. mexicana LPG and GPI8 mutant parasites to L. longipalpis}

We examined the binding of $L$. mexicana LPGnegative parasites to L. longipalpis midguts. In this series of the experiment we showed that the mutants bound less well than W'T controls, constituting only $25 \%$ of the total bound parasites (Table $1, P<0 \cdot 001$ ).

To show the key role of the other Leishmania surface molecules in parasite attachment to the sand fly midgut, competitive binding assays experiments using L. mexicana GPI8- and WT lines in L. longipalpis were performed. The GPI8 null mutant was generated by targeted disruption of the gpi8 gene that encodes the catalytic component of the GPI : protein transamidase complex, and does not express detectable levels of GPI-anchored proteins, including gp63, but have normal levels of other GPI-anchored molecules such as LPG and GIPLS (Hilley et al. 2000). Our results show that the WT line was found to account for over $99 \%$ of the parasites bound $(P<0 \cdot 001)$, indicating that WT parasites significantly out-competed GPI8 knockouts for binding sites ('Table 1).

\section{Binding of L. amazonensis P6.5/1.9 R mutant}

Gp63 is the most highly expressed protein on the surface of Leishmania promastigotes. To investigate its potential role, binding of the L. amazonensis gp63 down-regulated transfectant P6.5/1.9R to L. longipalpis midguts was investigated. This cell line bound in significantly lower numbers $(30 \% \pm 9$; $P<0.01$ ) than the control line $\mathrm{P} 6.5$ (mutant with vector alone) (Table 1$)$. This modest but statistically significant decrease suggests that the gp63 of L. amazonensis plays a role, albeit not critical, in the binding of its promastigotes to the L. longipalpis midgut.

\section{DISCUSSION}

This study provides data on the role of Leishmania surface glycoconjugates comparing results of two techniques: in vitro-binding experiments and in vivo development of Leishmania in sand flies. Firstly, we tested the binding of the L. major LPG mutant to $P$. papatasi midguts by the in vitro binding assay. The observed poor binding of the mutated line in comparison with the WT is consistent with the established observation for the dependency of L. major binding on LPG, as shown previously using other assays (Sacks et al. 2000; Kamhawi et al. 2004; Myskova et al. 2007). On the other hand, the same $L$. major mutated line bound as efficiently as the WT when incubated with the midguts of a permissive vector P. perniciosus. Phlebotomus perniciosus is the proven vector of $L$. infantum in the western Mediterranean (Killick-Kendrick, 1999) and according to previous data belongs to the group of permissive vectors (Volf and Myskova, 2007), which is susceptible for development of a wide range of Leishmania species. Our observation is in line with the results of in vivo experiments reported in earlier studies. Svarovska et al. (2010) showed that L. major LPG-mutants are able to complete their development in $P$. pernicious as well as the WT parasites. Similar results were observed from analysis of L. major in 2 other permissive species Phlebotomus argentipes (Svarovska et al. 2010) and L. longipalpis (Myskova et al. 2007; Secundino et al. 2010). Taken together, the use of the competitive binding assay in the current study confirmed the presence of an LPGindependent L. major-binding mechanism within the midgut of the permissive vector $P$. perniciosus and provides further strong support for the crucial role of LPG in the specific vector P. papatasi.

Interestingly, different results were observed in the case of L. infantum, the parasite species transmitted 
by $P$. perniciosus in nature. The observed poor development of LPG-deficient L. infantum as well as their poor binding in comparison with the WT suggests an important role for LPG in the binding and development of this Leishmania species. Although the experimental approach was different, these data are consistent with a previously reported role of LPG for Leishmania donovani attachment in $P$. argentipes (Sacks et al. 2000). Unfortunately, as there are no add-back lines available for both of the mentioned mutants (Sacks et al. 2000; this work), the results gained by $P$. perniciosus $-L$. infantum and $P$. argentipes $-L$. donovani combinations should be treated with caution; at present we cannot exclude the possibility that mutagenesis and prolonged cultivation in vitro also introduced changes in some non-target molecules.

Previous work with L. mexicana LPG-mutants demonstrated that these parasites were able to complete their development in L. longipalpis, although not as efficiently as W'T parasites (Rogers et al. 2004). The results of our in vitro binding study using this mutant line show that LPG of L. mexicana also plays some role in its binding to L. longipalpis midgut, although it appears to be less critical than for L. infantum in P. perniciosus or for L. major in P. papatasi.

In contrast to the intriguing data with $L$. infantum LPG mutant and P. perniciosus, it was not surprising to see that both the WT and the LPG-deficient $L$. infantum line failed to develop in the specific vector $P$. sergenti. The results obtained in vivo were clearly reflected in the poor in vitro binding of both L. infantum lines (WT line and LPG mutant) seen with $P$. sergenti midguts, which is in agreement with findings obtained by Killick-Kendrick et al. (1995) and Kamhawi et al. (2000) using other Leishmania species, confirming the specificity of $P$. sergenti for Leishmania tropica only.

Leishmania possess a number of cell surface glycoconjugates in addition to LPG that could function as ligands/receptors in midgut binding. The majority of these molecules are GPI-anchored glycoproteins (McConville and Ferguson, 1993; Ferguson, 1994), including gp63/leishmanolysin, PSP2/gp42 and membrane proteophosphoglycan (mPPG). To investigate whether this class of molecules may be involved in LPG-independent midgut binding, assays were performed with a L. mexicana GPI8 null mutant (Hilley et al. 2000). The GPI8mutant displayed normal growth in liquid culture, was capable of differentiating into replicating amastigotes within macrophages in vitro and was infective to mice (Hilley et al. 2000). These authors suggested that GPI-anchored proteins are not essential for parasite infectivity and survival in a mammalian host, but could play a role in a vector part of the life cycle. This suggestion is supported by the results of our study, in which we showed that GPI-anchored proteins are essential for in vitro binding of L. mexicana promastigotes to L. longipalpis midguts.

The gp63 has been described as a virulence factor with several different roles in the vertebrate host (Chaudhuri et al. 1989; Brittingham et al. 1995; Kulkarni et al. 2006). On the other hand, its function in the vector is still unclear. Data reported by Joshi et al. suggest that gp63 is not essential for development and survival of L. major in Phlebotomus duboscq $i$ and $P$. argentipes (Joshi et al. 1998, 2002). Hajmova et al. (2004) showed that the gp63 down-regulated transfectants L. amazonensis (P6.5/1.9R) developed at a lower rate and with lower parasite density during the early stage of development in L. longipalpis, suggesting that gp63 could protect promastigotes from degradation by the midgut digestive enzymes. Given the results described with the $L$. mexicana GPI mutant above, it is interesting to note that L. amazonensis is very closely related to L. mexicana (in the same species complex), and they are therefore likely to be using similar attachment mechanisms in L. longipalpis. In addition, the putative role of gp63/leishmanolysin in its interaction with its insect host is reinforced by the results of Sadlova et al. (2006) who observed that repeated passaging of Leishmania through sand flies leads to enhanced gp63 expression. To these observations we can now add a potential role of gp63 in Leishmania midgut attachment. Leishmania amazonensis gp63 downregulated transfectants bound slightly less efficiently than the control line to the midguts of L. longipalpis in our in vitro binding assay.

A role for gp63 in parasite attachment has previously been proposed also in trypanosomatids other than Leishmania. Pereira et al. (2009, 2010) showed that monoxenic Herpetomonas samuelpessoai and Leptomonas species produce a 63-kDa metallopeptidase that has similar biochemical/immunological properties to Leishmania gp63 and contributes to the parasite adhesion to Aedes aegypti guts or Aedes albopictus cell line $\mathrm{C} 6 / 36$.

In conclusion, the results of this study have confirmed the important role of Leishmania LPG in the interaction of $L$. major with its specific vector $P$. papatasi. They also confirm the existence of an LPG-independent mechanism of attachment for L. major in $P$. perniciosus, and that midgut binding is a necessary, albeit insufficient, for the generation of transmissible infections. However, the molecules involved in the binding of promastigotes in permissive vectors appear to be multiple, including LPG itself, gp63 and, importantly, other GPI-anchored proteins.

These molecules may interact with one another, and are likely to differ in importance and composition among different vector-parasite combinations. Thus what emerges from these data is a more complex and nuanced definition for a Leishmania-permissive sand fly. Nevertheless, some important general 
conclusions can be drawn. It appears that although an LPG-independent mechanism definitely exists, it is most clearly manifested when the midgut of a permissive sand fly is exposed to an unnatural parasite (L. major in $P$. perniciosus), whereas the natural parasite of the permissive sand fly may rely mainly, although not necessarily exclusively, on LPG for midgut binding (L. infantum in P. perniciosus). This has interesting evolutionary and epidemiological implications. It suggests that LPG-independent mechanism(s) could be advantageous for Leishmania in a chance-encounter with a new and permissive sand fly host by having multiple ligands alternative to LPG for evolutionary adaptation to survive in the midgut. The parasite may subsequently fine-tune its LPG for adaptation to evolve its utility as the main midgut binding ligand. This makes sense given that LPG is always the dominant cell surface glycoconjugate in Leishmania promastigotes. Thus, although multiplicity and complexity of the LPG-independent mechanisms render their analysis difficult they are important for further investigation as leishmaniasis continues to spread to new foci around the world.

\section{ACKNOWLEDGEMENTS}

We thank Jeremy Mottram for providing the L. mexicana GPI8 mutant. This work was supported by Project Grant 078937 from The Wellcome Trust; L. J., V. S. and P. V. were partially supported by EU grant 2011-261504 EDENext and the paper is catalogued by the EDENext Steering Committee as EDENext094.

\section{REFERENCES}

Bates, P. A. and Rogers, M.E. (2004). New insights into the developmental biology and transmission mechanisms of Leishmania. Current Molecular Medicine 4, 601-609.

Brittingham, A., Morrison, C. J., McMaster, W. R., McGwire, B.S., Chang, K. P. and Mosser, D. M. (1995). Role of the Leishmania surface protease gp63 in complement fixation, cell adhesion, and resistance to complement-mediated lysis. Fournal of Immunology 155, 3102-3111.

Brooks, D. R., McCulloch, R., Coombs, G.H. and Mottram, J.C. (2000). Stable transformation of trypanosomatids through targeted chromosomal integration of the selectable marker gene encoding blasticidin S deaminase. FEMS Microbiology Letters 186, 287-291.

Cihakova, J. and Volf, P. (1997). Development of different Leishmania major strains in the vector sandflies Phlebotomus papatasi and P. duboscqi. Annals of Tropical Medicine and Parasitology 91, 267-279.

Chaudhuri, G., Chaudhuri, M., Pan, A. and Chang, K. P. (1989). Surface acid proteinase (gp63) of Leishmania mexicana. A metalloenzyme capable of protecting liposome-encapsulated proteins from phagolysosomal degradation by macrophages. Fournal of Biological Chemistry 264, 7483-7489.

Chen, D. Q., Kolli, B. K., Yadava, N., Lu, H. G., Gilman-Sachs, A., Peterson, D. A. and Chang, K. P. (2000). Episomal expression of specific sense and antisense mRNAs in Leishmania amazonensis: modulation of gp63 level in promastigotes and their infection of macrophages in vitro. Infection and Immunity 68, 80-86.

Ferguson, M. A. (1994). What can GPI do for you? Parasitology Today 10, $48-52$.

Hajmova, M., Chang, K.P., Kolli, B. and Volf, P. (2004). Downregulation of gp63 in Leishmania amazonensis reduces its early development in Lutzomyia longipalpis. Microbes and Infection 6, 646-649.

Hilley, J. D., Zawadzki, J. L., McConville, M. J., Coombs, G. H. and Mottram, J. C. (2000). Leishmania mexicana mutants lacking glycosylphosphatidylinositol (GPI):protein transamidase provide insights into the biosynthesis and functions of GPI-anchored proteins. Molecular Biology of the Cell 11, 1183-1195.

Ilg, T., Demar, M. and Harbecke, D. (2001). Phosphoglycan repeatdeficient Leishmania mexicana parasites remain infectious to macrophages and mice. Fournal of Biological Chemistry 276, 4988-4997.

Joshi, P. B., Sacks, D. L., Modi, G. and McMaster, W. R. (1998). Targeted gene deletion of Leishmania major genes encoding developmental stage-specific leishmanolysin (GP63). Molecular Microbiology 27, 519-530.

Joshi, P.B., Kelly, B.L., Kamhawi, S., Sacks, D. L. and McMaster, W. R. (2002). Targeted gene deletion in Leishmania major identifies leishmanolysin (GP63) as a virulence factor. Molecular and Biochemical Parasitology 120, 33-40.

Kamhawi, S. (2006). Phlebotomine sand flies and Leishmania parasites: friends or foes? Trends in Parasitology 22, 439-445.

Kamhawi, S., Modi, G. B., Pimenta, P. F., Rowton, E. and Sacks, D. L. (2000). The vectorial competence of Phlebotomus sergenti is specific for Leishmania tropica and is controlled by species-specific, lipophosphoglycanmediated midgut attachment. Parasitology 121, 25-33.

Kamhawi, S., Ramalho-Ortigao, M., Pham, V. M., Kumar, S., Lawyer, P. G., Turco, S. J., Barillas-Mury, C., Sacks, D. L. and Valenzuela, J. G. (2004). Role for insect galectins in parasite survival. Cell 119, 329-341.

Killick-Kendrick, R. (1999). The biology and control of Phlebotomine sand flies. Clinics in Dermatology 17, 279-289.

Killick-Kendrick, R., Killick-Kendrick, M. and Tang, Y. (1995). Anthroponotic cutaneous leishmaniasis in Kabul, Afghanistan: the high susceptibility of Phlebotomus sergenti to Leishmania tropica. Transaction of Royal Society of Tropical Medicine and Hygiene 89, 477.

Kulkarni, M. M., McMaster, W.R., Kamysz, E., Kamysz, W., Engman, D. M. and McGwire, B.S. (2006). The major surfacemetalloprotease of the parasitic protozoan, Leishmania, protects against antimicrobial peptide-induced apoptotic killing. Molecular Microbiology 62, 1484-1497.

McConville, M. J. and Ferguson, M. A. (1993). The structure, biosynthesis and function of glycosylated phosphatidylinositols in the parasitic protozoa and higher eukaryotes. Biochemical fournal 294, 305-324.

Myskova, J., Svobodova, M., Beverley, S. M. and Volf, P. (2007). A lipophosphoglycan-independent development of Leishmania in permissive sand flies. Microbes and Infection 9, 317-324.

Pereira, F. M., Bernardo, P.S., Dias Junior, P.F., Silva, B. A., Romanos, M. T., d'Avila-Levy, C.M., Branquinha, M.H. and Santos, A. L. (2009). Differential influence of gp63-like molecules in three distinct Leptomonas species on the adhesion to insect cells. Parasitology Research 104, 347-353.

Pereira, F.M., Dias, F.A., Elias, C. G., d'Avila-Levy, C. M., Silva, C.S., Santos-Mallet, J.R., Branquinha, M.H. and Santos, A. L. (2010). Leishmanolysin-like molecules in Herpetomonas samuelpessoai mediate hydrolysis of protein substrates and interaction with insect. Protist 161, 589-602.

Rogers, M. E., Ilg, T., Nikolaev, A. V., Ferguson, M. A. and Bates, P. A. (2004). Transmission of cutaneous leishmaniasis by sand flies is enhanced by regurgitation of fPPG. Nature 430, 463-467.

Sacks, D. L. and Kamhawi, S. (2001). Molecular aspects of parasitevector and vector-host interactions in leishmaniasis. Annual Review of Microbiology 55, 453-483.

Sacks, D. L., Modi, G., Rowton, E., Späth, G., Epstein, L., Turco, S. J. and Beverley, S. M. (2000). The role of phosphoglycans in Leishmaniasand fly interactions. Proceedings of the National Academy of Sciences, USA 97, 406-411.

Sadlova, J., and Volf, P. (2009). Peritrophic matrix of Phlebotomus duboscqi and its kinetics during Leishmania major development. Cell and Tissue Research 337, 313-325.

Sadlova, J., Volf, P., Victoir, K., Dujardin, J. C. and Votypka, J. (2006). Virulent and attenuated lines of Leishmania major: DNA karyotypes and differences in metalloproteinase GP63. Folia Parasitologica (Praha) 53, 81-90.

Secundino, N., Kimblin, N., Peters, N. C., Lawyer, P., Capul, A. A., Beverley, S. M., Turco, S. J. and Sacks, D. (2010). Proteophosphoglycan confers resistance of Leishmania major to midgut digestive enzymes induced by blood feeding in vector sand flies. Cellular Microbiology 12, 906-918.

Späth, G. F., Epstein, L., Leader, B., Singer, S. M., Avila, H. A., Turco, S. J. and Beverley, S. M. (2000). Lipophosphoglycan is a virulence factor distinct from related glycoconjugates in the protozoan parasite Leishmania major. Proceedings of the National Academy of Sciences, USA 97, 9258-9263.

Svarovska, A., Ant, T.H., Seblova, V., Jecna, L., Beverley, S. M. and Volf, P. (2010). Leishmania major glycosylation mutants require 
phosphoglycans (lpg2-) but not lipophosphoglycan (lpg1-) for survival in permissive sand fly vectors. PLoS Neglected Tropical Diseases 4, e580.

Volf, P. and Myskova, J. (2007). Sand flies and Leishmania: specific versus permissive vectors. Trends in Parasitology 23, 91-92.

Volf, P. and Volfova, V. (2011). Establishment and maintenance of sand fly colonies. Fournal of Vector Ecology 36, Suppl. 1, S1-S9. doi: 10.1111/j.19487134.2011.00106.x.
Wilson, R., Bates, M. D., Dostalova, A., Jecna, L., Dillon, R. J., Volf, P. and Bates, P.A. (2010). Stage-specific adhesion of Leishmania promastigotes to sand fly midguts assessed using an improved comparative binding assay. PLoS Neglected Tropical Diseases 4, e816.

Yao, C., Donelson, J. E. and Wilson, M. E. (2003). The major surface protease (MSP or GP63) of Leishmania sp. Biosynthesis, regulation of expression, and function. Molecular and Biochemical Parasitology 132, 1-16. 